\title{
Parametric Instability of a Sandwich Beam with an Electrorheological Fluid Core Subjected to Various Boundary Conditions
}

\author{
Sukesh C. Mohanty \\ Department of Mechanical Engineering, National Institute of Technology, Rourkela, Orissa, 769008, India.
}

(Received 5 December 2008; revised 5 September 2009; accepted 7 September 2010)

\begin{abstract}
In the present study, the parametric instability of a three layer sandwich beam with an embedded electrorheological (ER) fluid core has been studied under various boundary conditions, namely fixed-fixed, pinned-pinned, fixed-free, and fixed-pinned. The beam has been modeled using finite elements and the regions of instability have been established using Saito and Otomi conditions. The ER core model is based on the pre-yield rheological properties and is represented by the complex shear modulus. The sandwich model is based on shear configuration. The effects of the electric field, shear parameter, and core thickness parameter on the fundamental frequency, fundamental buckling load, and fundamental system loss factor have been investigated. The effects of different parameters, such as the electric field, shear parameter, thickness parameter, and static load factor on the stability behavior of the beam have been investigated. Increasing the electric field strength, core thickness ratio, and shear parameter has a stabilizing effect for all the boundary conditions; by contrast, increasing the static load factor has a destabilizing effect for these support conditions.
\end{abstract}

\section{NOMENCLATURE}

$A_{k} \quad$ Cross-sectional area of the kth constraining elastic layer.

$A_{2} \quad$ Cross-sectional area of the ER fluid layer.

$L_{e} \quad$ Length of the beam element.

$t_{k} \quad$ Thickness of the $k^{\text {th }}$ constraining elastic layer, $k=1,3$.

$t_{2} \quad$ Thickness of the ER fluid layer.

$u_{k} \quad$ Axial displacement of the $k^{\text {th }}$ elastic layer, $k=1,3$.

$u_{2} \quad$ Axial displacement of the ER fluid layer.

$w \quad$ Transverse displacement of the beam.

$\alpha \quad$ Static load factor.

$\beta \quad$ Dynamic load factor.

$\gamma \quad$ Shear strain of the ER fluid layer.

$\{\Gamma\} \quad$ Set of generalized coordinates.

\section{INTRODUCTION}

Electrorheological (ER) fluids transform to a solid-like gel upon application of an electric field. The stiffness and damping depend on the magnitude of the applied voltage. Winslow first discovered ER fluid in 1949. ${ }^{1}$ Block et al. reported that the change in material properties is reversible and occurs in milliseconds. $^{2}$ These reversible changes are a result of the chain formation of micron-sized dielectric particles in nonpolar media under the applied electric field. When such chains are formed, the magnitudes of the rheological properties of the ER fluids change by several orders. Yield strengths of a typical ER fluid are of the order of $10 \mathrm{kPa}$ and $5 \mathrm{kPa}$ under static and dynamic loading conditions, respectively, for electric fields (both $\mathrm{AC}$ and DC) of the order of $3.5 \mathrm{kV} / \mathrm{mm}$ to $4.0 \mathrm{kV} / \mathrm{mm}$. Coulter reported the use of this innovative fluid in numerous engineering applications, including antivibration mounts, clutches, and dampers. ${ }^{3}$ Weiss et al. presented a summary of the state of research and development of ER fluid. ${ }^{4}$ Smart structures constructed with ER material can adapt to variable loading, reducing unwanted vibration. These structures are termed "semi- active" because the applied voltage changes the stiffness and damping coefficients in the governing equations of motion.

Sandwich beam-like structures with a viscoelastic material core have been extensively investigated to study the vibration controlling ability of viscoelastic materials. Kerwin was the first to carry out a quantitative analysis of the damping effectiveness of a constrained viscoelastic layer, and he obtained an expression to estimate the loss factor. ${ }^{5}$ Ungar derived general expressions for the loss factors of uniform linear composites in terms of the properties of the constituting materials. ${ }^{6}$ Di Taranto developed a theory to estimate natural frequencies, loss factors for a finite length sandwich beam. ${ }^{7}$ Mead and Markus carried out the forced vibration analysis of a threelayer sandwich beam with a viscoelastic core and with arbitrary boundary conditions. ${ }^{8}$ They followed the method used in an analysis conducted by Di Taranto. Rao included graphs and equations in his work to estimate frequencies and loss factors for sandwich beams under various boundary conditions. ${ }^{9}$ Asnani and Nakra carried out forced vibration analysis of sandwich beams with viscoelastic cores and with fixedfixed and cantilever-type end conditions. ${ }^{10}$ The forced vibration response obtained by applying the Ritz method matched well with their experimental results. Johnson and his coworkers used the finite element method to solve frequencies and loss factors for beams and plates with constrained viscoelastic layer. ${ }^{11,12}$ Bhimaraddi solved both the resonant frequencies and loss factors for a simply supported beam with constrained layer damping using a model that accounted for the continuity of displacements and the transverse shear stresses across the interfaces of the layers. ${ }^{13}$ Banerjee studied the free vibration of a three-layer sandwich beam using the dynamic stiffness matrix method. ${ }^{14} \mathrm{He}$ calculated the natural frequencies and mode shapes. Sun et al. developed a finite element model to study the effect of an add-on viscoelastic layer in damping and vibration control of unidirectional composite laminates. ${ }^{15}$ 